\title{
Laser-induced acoustic wave generation/propagation/interaction in water in various internal channels
}

\author{
Seung Hwan Ko • Daeho Lee $\cdot$ Heng Pan • \\ Sang-Gil Ryu • Costas P. Grigoropoulos • Nick Kladias • \\ Elias Panides • Gerald A. Domoto
}

Received: 28 May 2010 / Accepted: 1 June 2010 / Published online: 30 June 2010

(C) The Author(s) 2010. This article is published with open access at Springerlink.com

\begin{abstract}
Short pulsed laser-induced single acoustic wave generation, propagation, interaction within a water-filled internal channel are experimentally and numerically studied. A large-area, short-duration, single-plane acoustic wave was generated by the thermoelastic interaction of a homogenized nanosecond pulsed laser beam with a liquid-solid interface and propagated at the speed of sound in water. Laser flash Schlieren photography was used to visualize the transient interaction of the plane acoustic wave in various internal channel structures ((a) sudden expansion and contraction channels, (b) bifurcating channels, (c) gradual contraction wall channels and (d) a cylinder). Fairly good agreement between the experimental results and numerical simulation is observed.
\end{abstract}

\section{Introduction}

The short pulsed laser-induced acoustic wave generation and detection in liquid and solid media have been demonstrated to be efficient tools in many applied science and medical areas, including nondestructive evaluation and material characterization [1-3], laser cleaning of surface contaminants [4], laser tissue ablation, corneal sculpturing [5],

\section{S.H. Ko $(\bowtie)$}

Department of Mechanical Engineering, KAIST, Daejeon, Korea

e-mail: maxko@kaist.ac.kr

D. Lee $\cdot$ H. Pan · S.-G. Ryu · C.P. Grigoropoulos $(\bowtie)$

Department of Mechanical Engineering, University of California, Berkeley, CA 94720, USA

e-mail: cgrigoro@me.berkeley.edu

N. Kladias · E. Panides · G.A. Domoto

Xerox Research Center Webster (XRCW), 141 Webber Avenue,

Sleepy Hollow, NY 10591, USA and gall stone fragmentation [6]. The laser energy interaction with the surface of an absorbing liquid or a transparent liquid in contact with an absorbing solid boundary induces rapid heating, thermoelastic expansion or explosive phase change and finally emission of a strong ultrasonic wave or a shock wave, depending on the applied laser energy [7, 8].

Most of the laser-induced acoustic wave experimental studies have aimed at the zero-dimensional point detection of the propagating pressure transient at a fixed location by utilizing capacitance transducers [1], piezoelectric transducers [9], or hydrophones [10]. These approaches can only be applied to idealized zero- or one-dimensional geometries and fail to capture the detailed acoustic wave behavior inside the medium, especially for complex two- or three-dimensional configurations. Several numerical simulation studies were carried out to discuss multidimensional laser-induced acoustic wave behavior. Ko et al. first reported the time-resolved direct observation of two- or threedimensional laser-induced acoustic wave interaction with submerged solid structures such as single, double, $33^{\circ}$ tilted single block, and concave cylindrical acoustic lens configurations [11, 12]. They further demonstrated that these waves can be focused down to a domain of several tens of microns in size and acoustic focusing lens has potential applications to biomaterial processing and characterization. Compared with submerged structures, acoustic wave propagation and interaction in internal channels are more frequently found in many practical applications such as in an inkjet head (gradual or sudden contraction wall channel) and bifurcating coronary artery (T-branched and Y-branched channels).

In this paper, we present time resolved imaging of laser-induced single, short acoustic plane wave propagation in various water-filled "internal" channel configurations: (a) sudden expansion and contraction channels, (b) bifurcating channels: T-branched and Y-branched (bifurcating coro- 
nary artery) channels, (c) gradual contraction wall channels: linear contraction and parabolic contraction (inkjet nozzle) wall channel, (d) a cylinder. Experimental observations were compared with finite element method (FEM) based numerical simulations.

\section{Experiment and numerical simulations}

The generation/propagation/interaction of laser-induced acoustic waves in internal channels were directly observed by two laser systems with different wavelength. The two laser systems were combined to achieve different functions: (1) neodymium-doped yttrium aluminum garnet (Nd:YAG) nanosecond pulsed laser with green wavelength $(532 \mathrm{~nm})$ to generate the acoustic wave and (2) nitrogen laser pumped dye laser with blue wavelength $(440 \mathrm{~nm})$ as an illumination source for the time resolved acoustic wave imaging. The two lasers were synchronized via a delay generator to control the precise time delay between the two laser pulse firings [13]. All images went through background correction image processing to reduce background noise. Besides the experimental observations, numerical simulations were carried out to compare with experiment and also to further explain detailed physics.

\subsection{Time resolved acoustic wave imaging: two-laser synchronization}

Figure 1(a) shows how two lasers were combined and synchronized to acquire time resolved images of the laserinduced acoustic wave propagation in water and interaction with internal channel walls. A frequency-doubled, homogenized Nd:YAG pulsed laser beam [indicated by green colored path, New Wave Inc., wavelength $=532 \mathrm{~nm}$, temporal pulse width $=5 \mathrm{~ns}$ full width half maximum (FWHM)] was applied normal to the vacuum deposited 100-nm-thick chromium (Cr) film on a $800-\mu$ m-thick fused quartz substrate. The Nd:YAG laser is first absorbed by the $\mathrm{Cr}$ thin film directly attached to the de-ionized (DI) water-filled channels. Upon laser-induced heating, both the liquid and the $\mathrm{Cr}$ thin film experience thermoelastic expansion and stress fields, and thereby launch an acoustic wave into optically and acoustically transparent water. The Nd:YAG laser beam cross section was shaped to a $2 \times 2 \mathrm{~mm}^{2}$ flat-top beam profile of very good spatial uniformity by a microlens laser beam homogenizer [SUSS MicroOptics] combined with a $10 \times$ long working distance objective lens [Mitutoyo]. The $\mathrm{Nd}$ :YAG energy fluence applied to induce pressure wave was around $12-14 \mathrm{~mJ} / \mathrm{cm}^{2}$ which is substantially lower than the Cr melting threshold $\left(120 \mathrm{~mJ} / \mathrm{cm}^{2}\right)$ [14] and even smaller than the bubble nucleation threshold $\left(36.5 \mathrm{~mJ} / \mathrm{cm}^{2}\right)$ [5]. For the laser energy levels considered in this work, transient laser-induced pressure waves observed are attributed solely to the thermoelastic response of the metal thin film and the adjacent liquid. Rapid bubble growth, and collapse, as well as plasma formation are not likely to contribute [4].

At a certain time delay after the firing of the Nd:YAG laser beam inducing acoustic wave, the nitrogen laser pumped dye laser beam [indicated by blue colored path, wavelength $=440 \mathrm{~nm}$, pulse width $=10 \mathrm{~ns}$ FWHM, Laser Science Inc.] was expanded and applied parallel to the metal thin film surface as a synchronized illumination light source. A short pass filter was placed in front of the zoom lens to block the Nd:YAG laser beam and to accept only the nitrogen laser pumped dye laser beam. Upon encountering pressure-induced refractive index variations by Nd:YAG laser beam, the imaging laser beam (nitrogen laser pumped dye laser beam) bends from the original beam path. Time resolved frozen images of the acoustic waves were acquired via this mechanism.
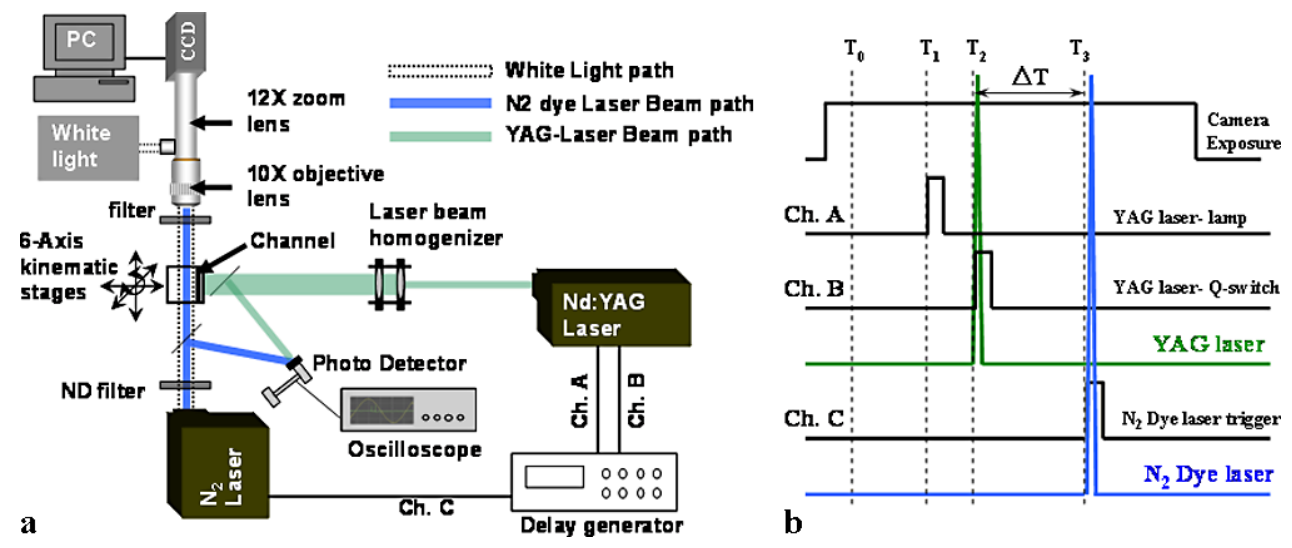

Fig. 1 (a) Schematic diagram of experimental setup for a laserinduced acoustic wave generation with a homogenized Nd:YAG laser (wavelength: $532 \mathrm{~nm}$, indicated by green colored path) and for the acoustic wave time resolved observation by a laser flash Schlieren photography with a delayed nitrogen laser pumped dye laser illumination (wavelength: $440 \mathrm{~nm}$, indicated by blue colored path). (b) Laser timing for time resolved Schlieren photography 
The temporal interval between the processing Nd:YAG and the illuminating nitrogen laser pumped dye laser beams was controlled by a delay generator [Stanford Research Systems] as shown in Fig. 1(b). Nd:YAG laser (indicated by a green colored line) was fired by two signals: lamp signal from channel $1(\mathrm{Ch} 1)$ at time $1\left(T_{1}\right)$ and Q-switch signal from channel $2(\mathrm{Ch} 2)$ at time $2\left(T_{2}\right)$. Nitrogen laser pumped dye laser illumination light source (indicated by a blue colored line) was fired by one trigger signal from channel 3 (Ch3) at time $3\left(T_{3}\right)$. The time delay $(\Delta T)$ between $T_{2}$ and $T_{3}$ determines the flight time of the acoustic wave after it was launched from the $\mathrm{Cr}$ surface. The actual time delay was measured with a photodetector [Thorlab] and an oscilloscope [Agilent].

All images went through background correction image processing by subtracting a background image from the pressure wave images. This processing was done with image analysis software [ImagePro Plus].

\subsection{Numerical simulations}

With the aid of Comsol Multiphysics 3.4a acoustics module, finite element method (FEM) based numerical simulations were performed to compare with the experimental results. The acoustic pressure, $p(\mathbf{r}, t)$ in a stationary lossless medium is governed by the following partial differential equation [15]:

$\frac{1}{\rho_{0} c_{s}^{2}} \frac{\partial^{2} p}{\partial t^{2}}+\nabla \cdot\left(-\frac{1}{\rho_{0}}(\nabla p)\right)=\mathbf{Q}$

where $\rho_{0}$ is the density $\left(\mathrm{kg} / \mathrm{m}^{3}\right), c_{s}$ the speed of sound $(\mathrm{m} / \mathrm{s})$, $\mathbf{Q}$ the source $\left(1 / \mathrm{s}^{2}\right)$. The combination $\rho_{0} c_{s}^{2}$ is called the adiabatic bulk modulus, commonly denoted by $K$. The density and speed of sound are assumed to be constant ( $\rho_{0}=$ $998 \mathrm{~kg} / \mathrm{m}^{3}, c_{s}=1481 \mathrm{~m} / \mathrm{s}$ ) because they vary with time on scales much larger than the characteristic acoustic wave period [15]. Sound-hard boundary was employed to model the channel surfaces (Fig. 2(b)-(i)\&(iii)), where the normal component of the particle velocity vanishes. As there is no acoustic drift velocity, this condition is equivalent to the normal acceleration being zero. For constant fluid density and zero source $\left(\mathbf{Q}=0 \mathrm{~s}^{-2}\right)$, this also means that the normal derivative of the pressure is zero at the boundary [15]:

$\frac{\partial p}{\partial n}=0$

Radiation boundary condition was applied to model open boundaries (Fig. 2(b)-(ii)). This condition allows the outgoing wave to leave the modeled domain with no or minimal reflection. The radiation boundary condition for tran-

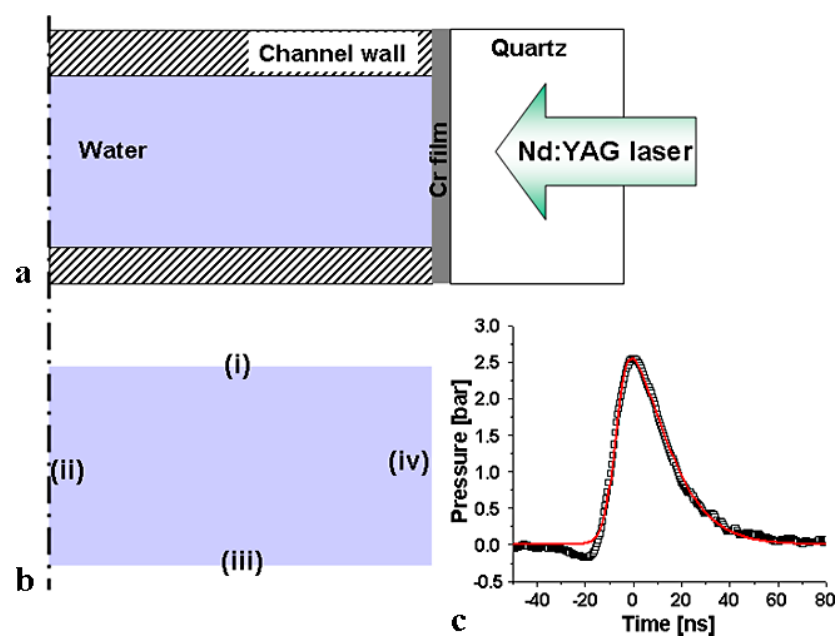

Fig. 2 (a) Internal channel configuration for experiment, (b) computation domain and boundary condition, (c) measured (black square sym$b o l)$ and best fitted (red solid line) laser-induced acoustic wave

sient analysis is the first-order expression [15]:

$$
\begin{aligned}
\mathbf{n} & \cdot\left(\frac{1}{\rho_{0}}(\nabla p)\right)+\frac{1}{\rho_{0}}\left(\frac{1}{c_{s}} \frac{\partial p}{\partial t}\right) \\
= & \frac{1}{\rho_{0}}\left(\frac{1}{c_{s}} \frac{\partial p_{0}}{\partial t}+\kappa(r) p_{0}+\mathbf{n} \cdot \nabla p_{0}\right)
\end{aligned}
$$

where $p_{0}$ describes an incident wave, $p_{0}(\mathbf{r}, t)$. Pressure boundary condition (Fig. 2(b)-(iv)) was applied to model initial laser-induced pressure field near the $\mathrm{Cr}$ thin film. The transient pressure source was assumed to follow the detected pressure (Fig. 2(c), square symbol) and the following bestfitted asymmetric double sigmoidal form (Fig. 2(c), red line) was assigned as the initial pressure boundary condition exerted by the $\mathrm{Cr}$ thin film:

$$
P[\mathrm{bar}]=\frac{4.12}{\left(1+\exp \left(-\frac{t+6.97}{2.51}\right)\right)}\left(1-\frac{1}{1+\exp \left(-\frac{t-6.97}{10.83}\right)}\right) .
$$

\section{Results and discussions}

Time resolved laser-induced acoustic wave images of plane acoustic wave propagation and behavior in water inside various channel configurations are presented in Figs. 3-7, juxtaposed with the numerical simulation results. The internal channels of interest in this study include various configurations: (a) sudden expansion and contraction channels, (b) bifurcating channels: T-branched and Y-branched channels, (c) gradual contraction wall channels: linear contraction and parabolic contraction wall channel, (d) a cylinder: trapped acoustic wave in a cylinder. 


\subsection{Sudden expansion and contraction channels}

In a sudden contraction channel (Fig. 3(a)), the middle part of the flat acoustic wave kept proceeding along the narrow channel while the side parts reflected upstream. The middle advancing part of the acoustic wave had sharp edges in the proximity of the narrow channel walls while the reflected acoustic wave induced a diverging cylindrical component due to diffraction at the sharp entrance of the contraction channel.

In the case of the sudden expansion channel (Fig. 3(b)), the incoming acoustic wave in a narrow channel maintained a flat profile flanked by cylindrical waves caused by diffraction near the sudden expansion edges. The cylindrical waves propagate both outwards and inwards. The outward expanding cylindrical acoustic waves attain a dove- tail shaped acoustic wave followed by secondary expanding dovetail acoustic waves from two inward expanding cylindrical acoustic waves. For both cases, numerical simulation showed fairly good agreement with experimental observation.

\subsection{Bifurcating channels: T-branched and Y-branched channels}

Two bifurcating channel configurations were studied; a Tbranched bifurcating wall channel and a Y-branched gradually bifurcating channel to simulate the pressure wave behavior at the coronary artery bifurcation.

The T-branched bifurcating channel case (Fig. 4) is the inverse geometry of the sudden contraction channel. However, interestingly, after the middle acoustic wave part
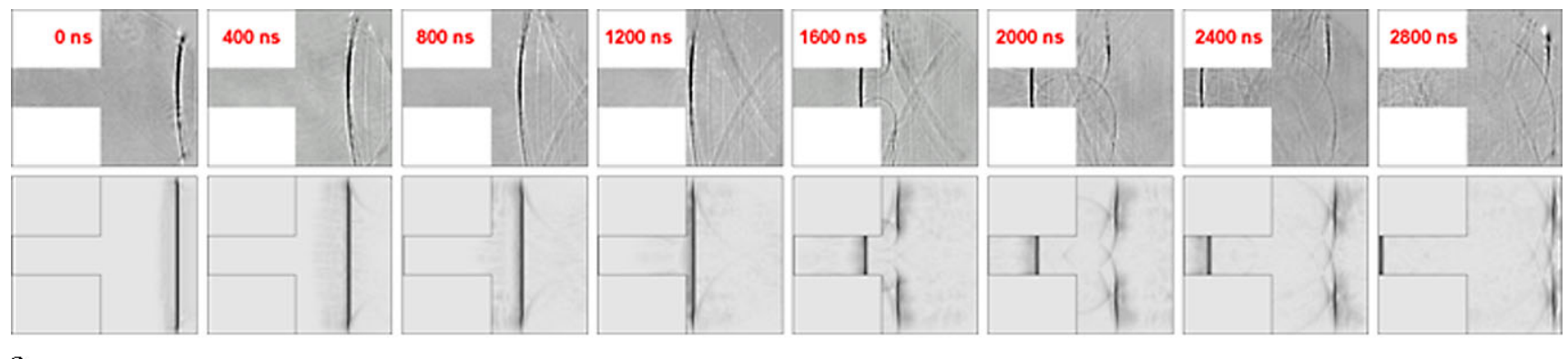

a
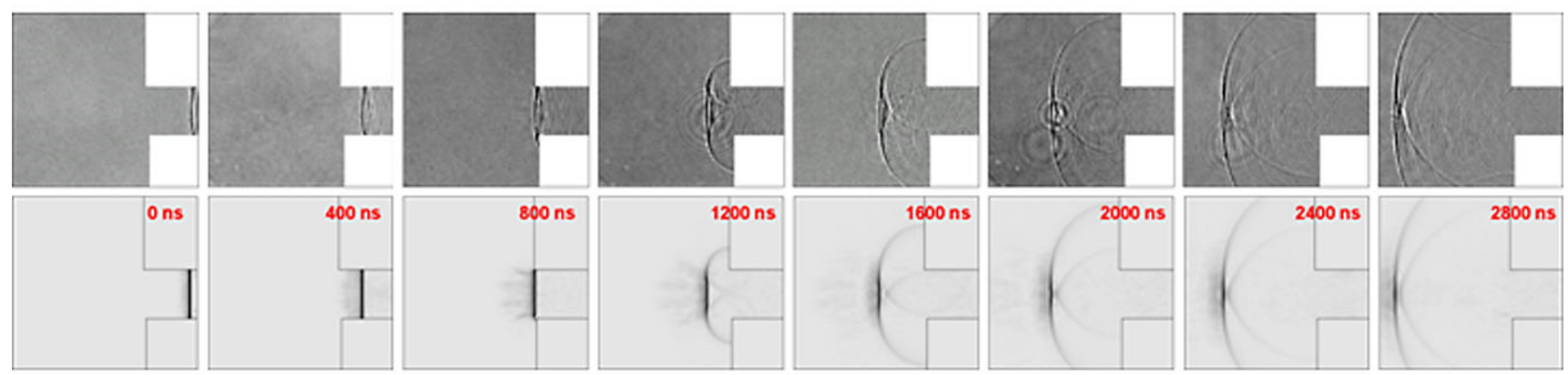

b

Fig. 3 (a) Sudden contraction channel and (b) sudden expansion channel (top rows: experiment; bottom rows: simulation)
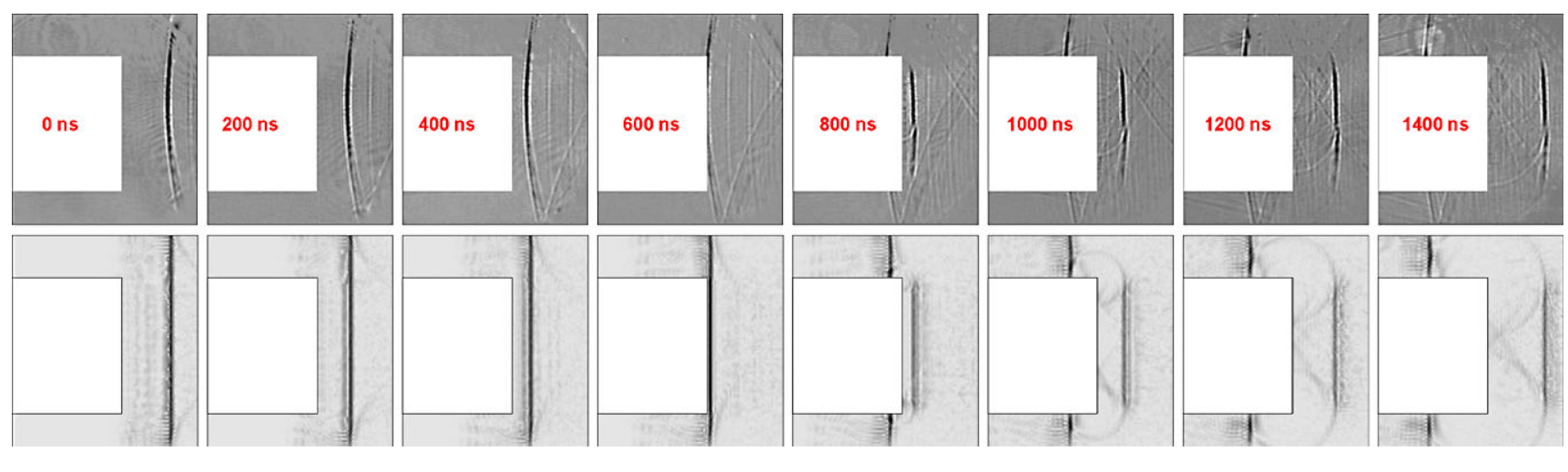

Fig. 4 T-branched bifurcating channel (top row: experiment; bottom row: simulation) 
bounded back, the reflected wave behaved exactly as in the sudden expansion channel case forming a double expanding dovetail cylindrical acoustic wave. The side parts of the incoming flat acoustic wave propagated unobstructed with sharp edges across the wall interfaces.

In the Y-branched gradually bifurcating channel case (Fig. 5), the incoming flat acoustic wave was bisected upon meeting the sharp tip edge. The halved acoustic waves were first reflected specularly on the $45^{\circ}$ tilted inner wall surface. The first reflected flat acoustic wave front moved upwards (vertically) and then reflected back to left (horizontally) at the outer wall and kept propagating along the channel down- stream via multiple reflections. The propagation behavior in this bifurcating channels can be exactly predicted from multiple reflections.

In both cases, numerical simulations showed fairly good agreement with the experimental observations.

\subsection{Gradual contraction wall channels: linear contraction and parabolic contraction wall channel}

Gradually contracting channels with both linear and parabolic contractions channel were studied to simulate the pressure wave interaction and behavior inside the inkjet nozzle.
Fig. 5 Y-branched bifurcating channel (left two columns: experiment; right two columns: simulation)
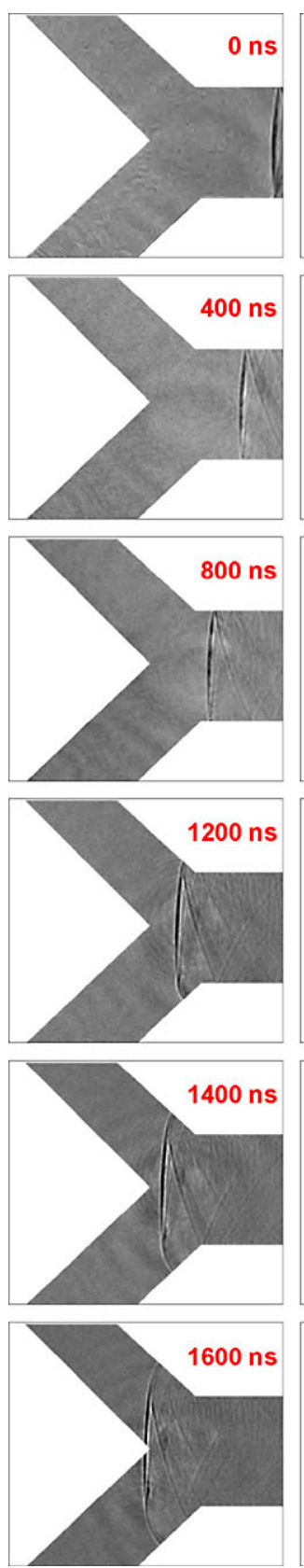
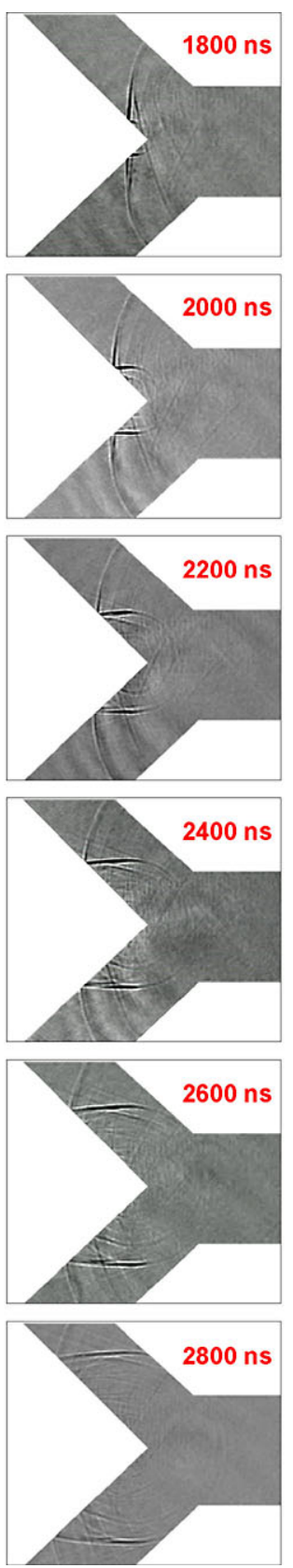
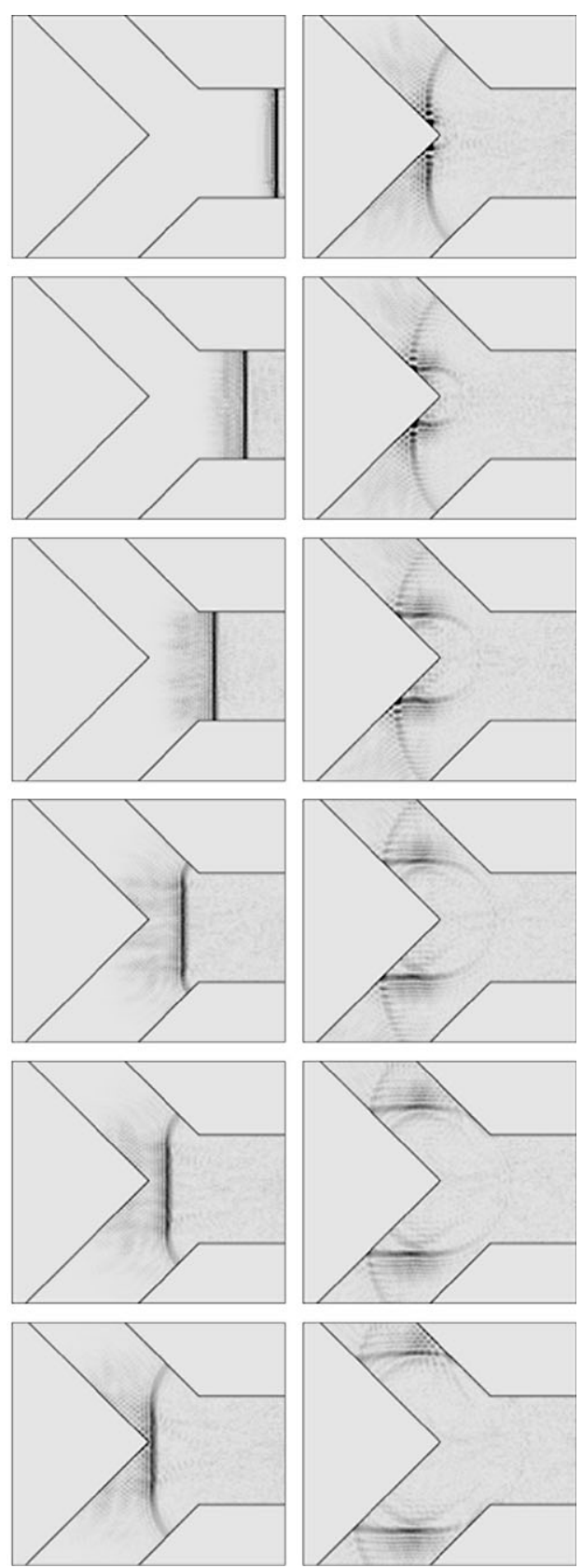
Fig. 6 (a) Linearly varying contraction wall and (b) parabolic contraction wall (left two columns: experiment; right two columns: simulation)
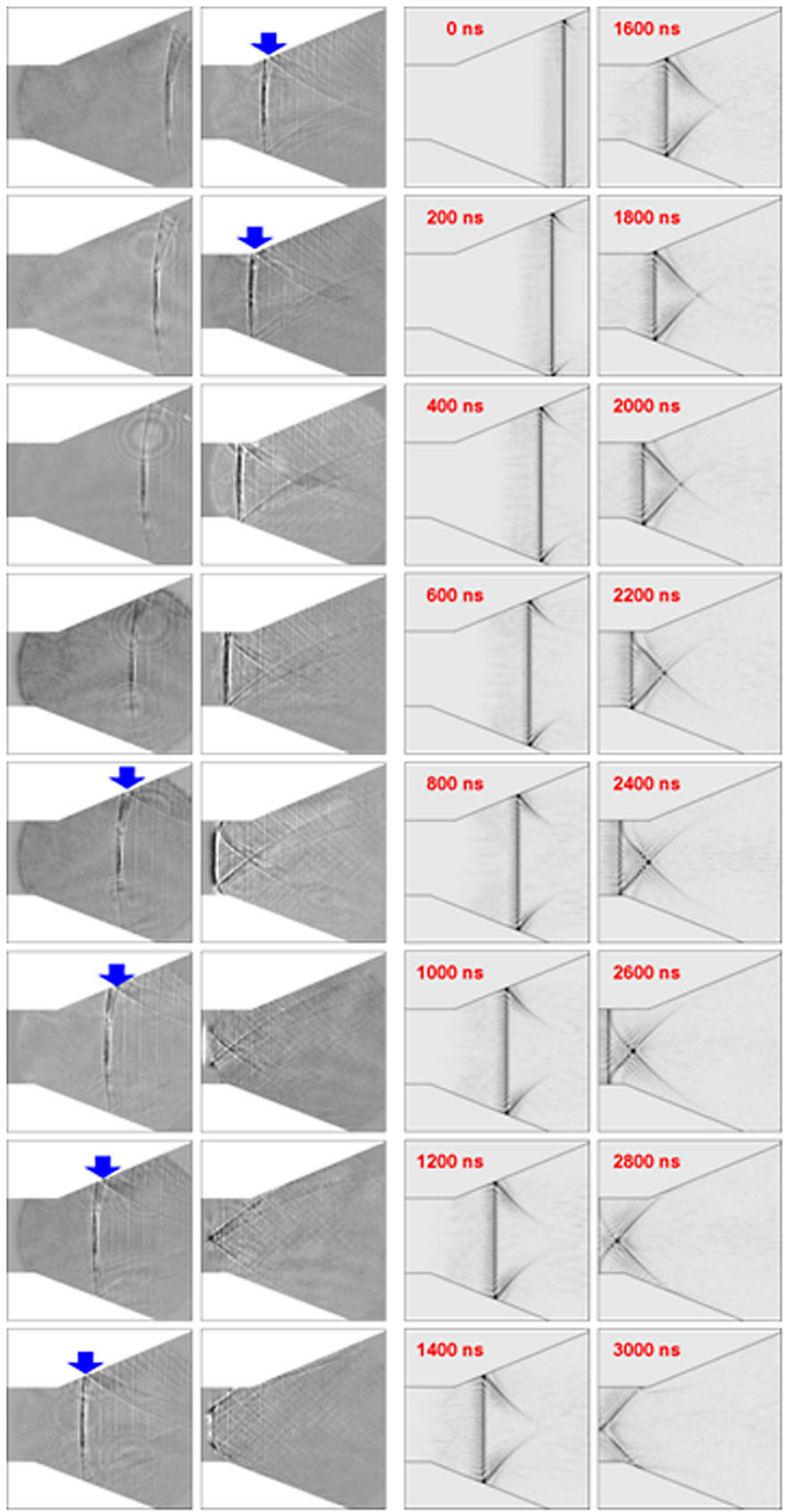

a

The jetting phenomenon in the piezoelectrically driven inkjet head has been known to be strongly related to the pressure wave inside the printhead [16]. However, in many cases, it was difficult to observe the pressure wave directly and there have been scarcely any reports in the open literature [17]. Consequently, a CCD camera with a strobe LED 
Fig. 6 (Continued)
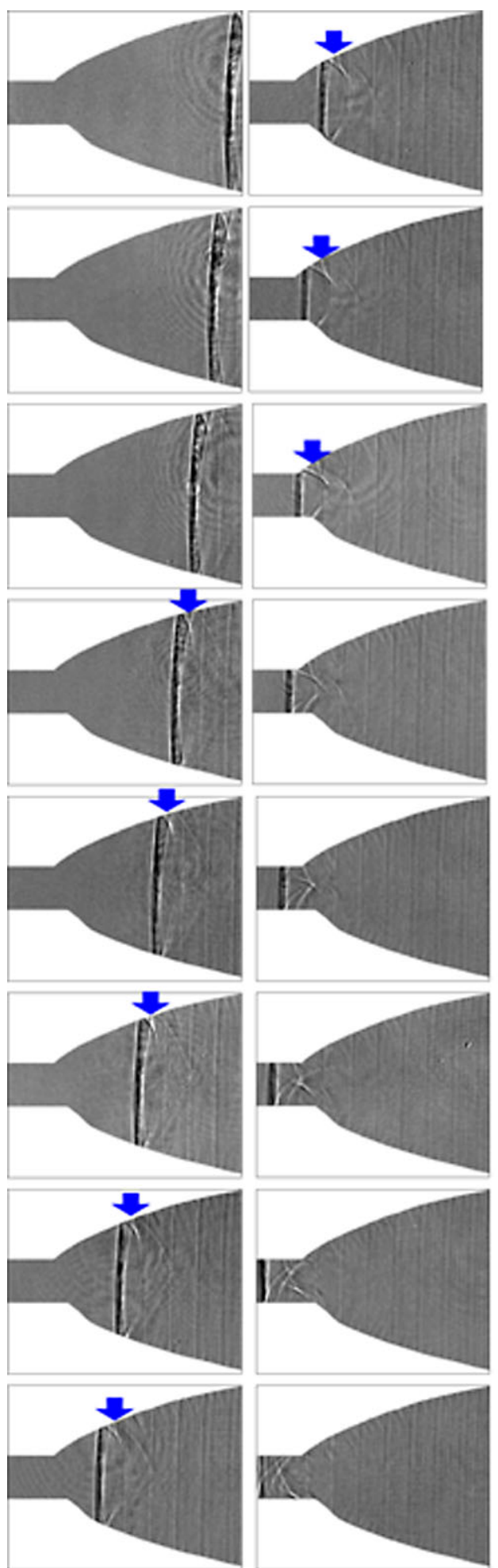

b
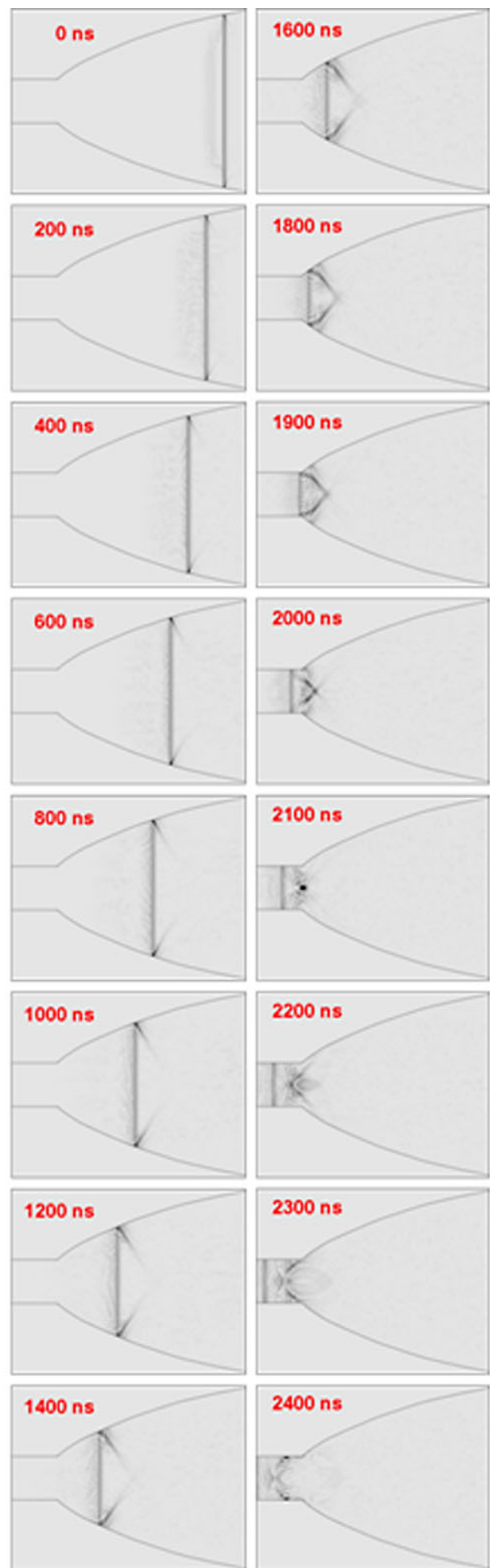

illumination was typically employed to measure the droplet speed or volume, instead of directly measuring the pressure wave inside the dispenser. However, it is hard to understand the pressure wave behavior inside the dispenser by just observing the droplet image [17]. Here, we conducted a direct observation of the pressure wave propagation and interaction inside nozzle geometry.

In the converging nozzle with straight wall (Fig. 6(a)), the reflection was specular at an angle of $30^{\circ}$. As the central part of the original pressure wave approached the end of the 
nozzle, both ends of the reflected pressure wave extended to long wings. Part of the reflected and extended pressure wave did not make it to the nozzle tip but reflected back. This implies that in this nozzle wall geometry, only a small portion of the pressure wave can reach the nozzle tip to contribute to the droplet formation while the rest of the pressure wave just reflects back and forth multiple times inside the capillary tube to finally dissipate. The slope of the nozzle wall may modulate the amplitude of the pressure at the nozzle tip. Nozzles with larger slope may enhance the pressure amplitude via a slight focusing effect, but the back reflection will also increase. On the other hand, nozzles with smaller slope do not impart focusing and experience little reflection.

In the converging nozzle with a parabolic wall (Fig. 6(b)), the flat acoustic wave also reflected against the walls. However, the reflected pressure waves behaved totally differently compared to the nozzle with a linearly varying slope

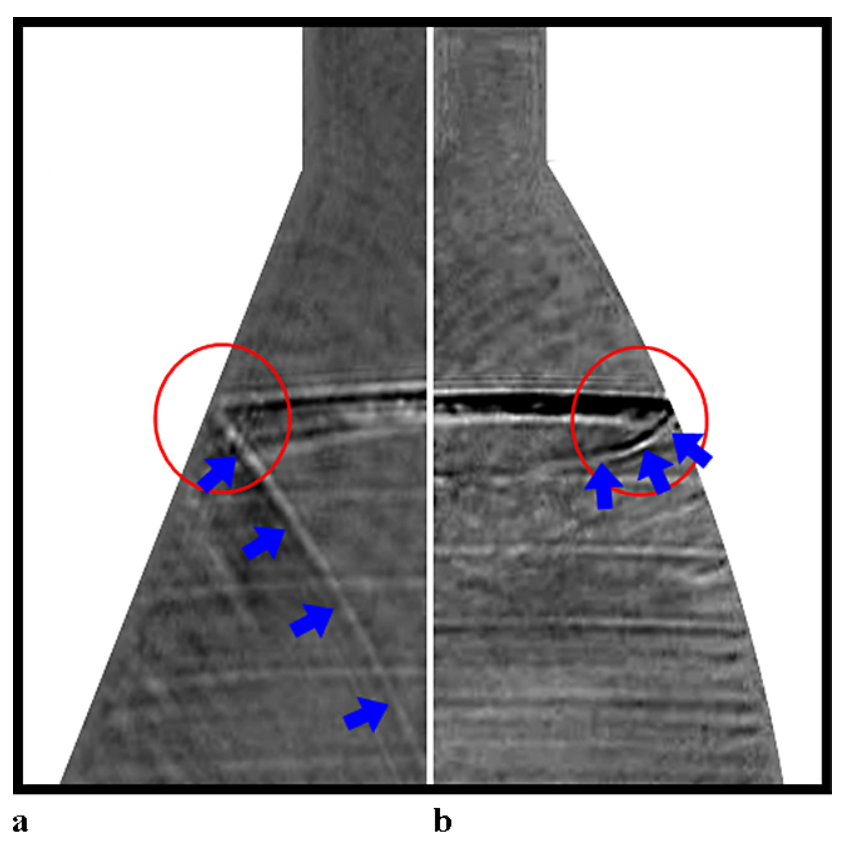

Fig. 7 Magnified view from Fig. 6 for reflected pressure wave at the nozzle wall for (a) linearly varying contraction wall and (b) parabolic contraction wall
(Fig. 6(a)). As the pressure wave entered the nozzle zone with small wall curvature, the reflection had a similar pattern to that previously examined in the nozzle with a linearly varying slope. As the pressure wave propagated toward the nozzle tip region where the curvature was higher, the pressure wave exhibited focusing near the nozzle wall. The focusing point was maintained near the nozzle wall and carried most of the concentrated pressure wave energy by suppressing the continuous expansion of the pressure wave after reflection from the nozzle wall that was previously observed in the linearly varying nozzle (Fig. 7). Our previous report [12] showed that the reflected pressure wave from a perfect cylindrical concave surface could be focused to a line $R / 2$ distant from the cylinder wall after the reflection. A similar focusing effect is proposed to explain the local focusing effect of the reflected pressure wave on the curved nozzle wall. However, if the nozzle wall has a non-zero constant curvature like a perfect cylinder (Fig. 8), the focus is not kept near the nozzle wall is rather fixed at $R / 2$ and to the right of the nozzle wall. This means that a nozzle with one fixed curvature (such as cylinder) will produce a highly focused pressure point although this is due to the back reflected pressure wave. After the focusing, the pressure wave will reflect back to the incoming direction and will be dissipated without contributing to the droplet generation, just like in the linearly varying nozzle case. More sophisticated nozzle wall shape is needed for the locally focused pressure wave to be confined near the nozzle wall and carried to the nozzle tip. To keep the focused pressure wave near the nozzle wall, the nozzle must have an increasing curvature towards the nozzle tip instead of having a fixed curvature.

Maximum pressure at the nozzle tip is implemented by carrying the concentrated pressure wave to the nozzle tip and minimizing the pressure wave back reflection loss. First, the nozzle wall should have a non-zero curvature (curved surface) imparting focusing effect to minimize the extension of the reflected pressure wave and backward reflection. Second, the nozzle must have a varying (increasing) curvature to keep the reflected wave focused point near the nozzle wall
Fig. 8 Pressure wave for perfect circular wall nozzle

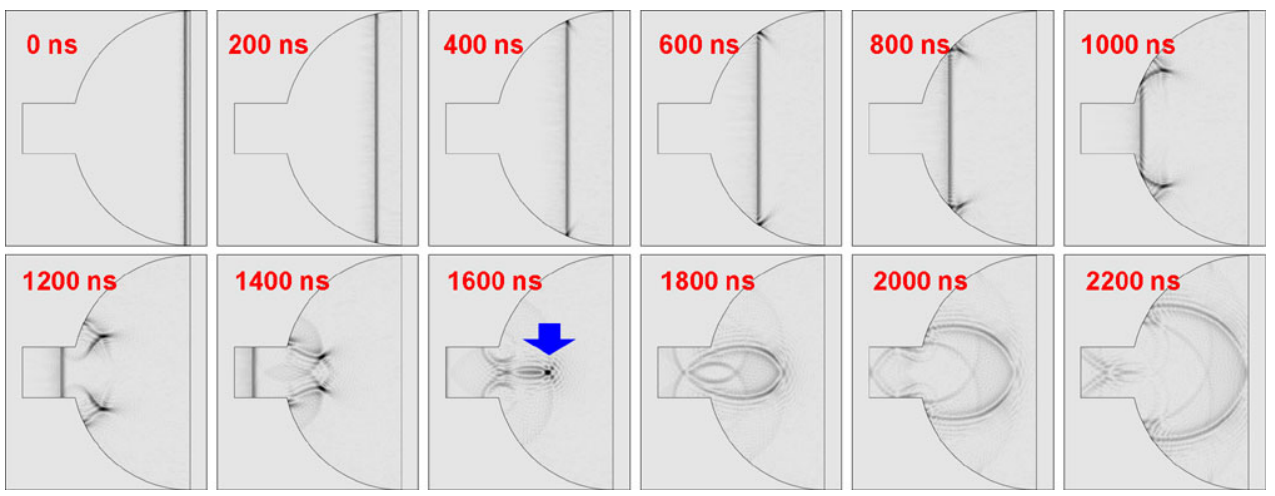


Fig. 9 Trapped acoustic wave in a cylinder (first and third rows: experiment; second and fourth rows: simulation)
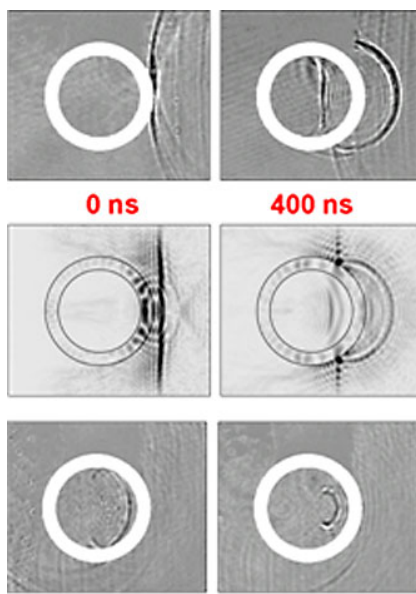

$1900 \mathrm{~ns}$

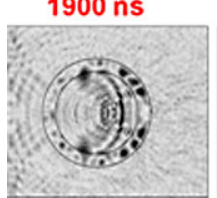

$400 \mathrm{~ns}$
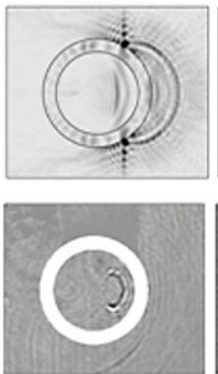

2200 ns

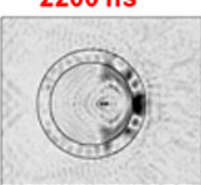

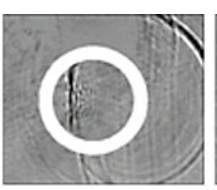

800 ns
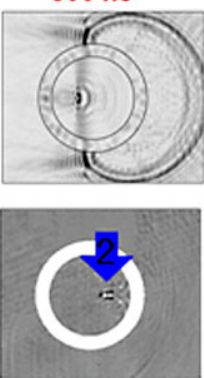

$2400 \mathrm{~ns}$

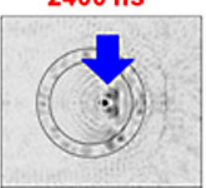

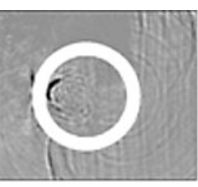

$1100 \mathrm{~ns}$

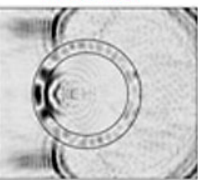

$1300 \mathrm{~ns}$
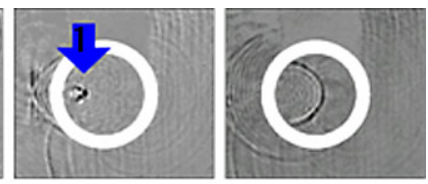

$1600 \mathrm{~ns}$
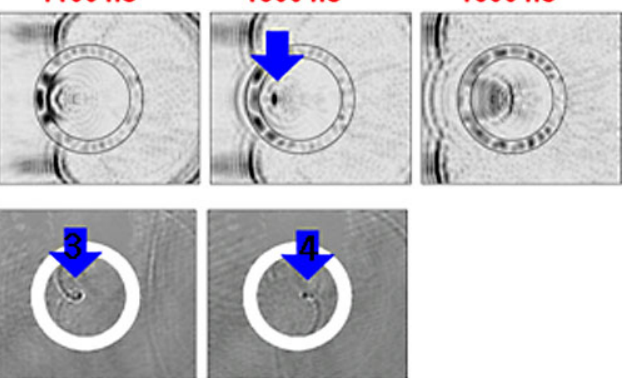

3500 ns
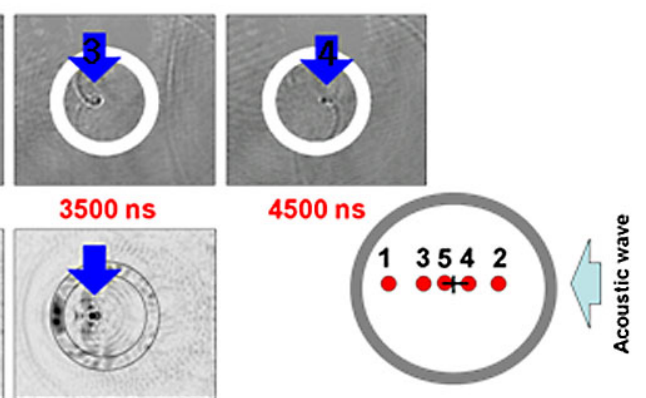

and bring the locally focused pressure wave to the tip of the nozzle with minimal back reflection.

The numerical simulations showed fairly good agreement with the experimental observations.

\subsection{Trapped acoustic wave in a cylinder}

A plane acoustic wave was launched against a cylinder (Fig. 9) in order to investigate trapped acoustic wave behavior within an enclosed geometry. First, the incoming plane acoustic wave was partially transmitted through the glass cylinder shell in part reflected toward the opposite direction diverging to a cylindrical wave. The transmitted acoustic wave propagated through the cylindrical enclosure maintaining a flat profile until it hit the opposite internal wall surface. The first reflected acoustic wave on the left inner cylinder wall focused in the left half of the cylinder (point "1" in Fig. 9). This wave, diverged after focusing and after reflection on the right inner wall, was focused again in the right half of the cylinder (point "2" in Fig. 9). These reflection-focusing events inside the cylinder repeat until the acoustic wave totally dissipates. During this reflectionfocusing process, the focusing point alternates (left-rightleft, and so on) and approaches the geometric center of the cylinder.

Acoustic wave inside the cylinder was studied to as an analog to the pressure wave interaction and behavior inside the human eyeball during the LASIK (laser-assisted in situ keratomileusis), a type of refractive surgery for correcting myopia, hyperopia, and astigmatism. The observation of the presence of the focusing point inside the cylinder suggests the possibility of high pressure generation.

The numerical simulations showed fairly good agreement with the experimental observations.

\section{Summary}

Experimental and numerical investigation on laser-induced acoustic wave generation, propagation, interaction in various internal channels filled with water are presented. A homogenized Nd:YAG laser beam (532 nm, $4 \mathrm{~ns}$ pulse width) was irradiated on a $\mathrm{Cr}$ thin film on a quartz substrate. A sharp plane acoustic wave was generated by laser-induced thermoelastic stress, and propagated at the speed of sound in water. Time resolved laser flash Schlieren photography images captured the acoustic wave propagation in the water and interaction with internal wall configurations, including (a) sudden expansion and contraction channels, (b) bifurcating channels, (c) gradual contraction wall channels and (d) a hollow cylinder. The experimental results showed fairly good agreement with numerical simulations.

Acknowledgements This work was financially supported by the University of California, Berkeley by Xerox Wilson Center for Research and Technology, the Ministry of Knowledge Economy of Korea under Grant No. 10032145, and by National Research Foundation (NRF) of Korea under Grant No. 2010-0003973.

Open Access This article is distributed under the terms of the Creative Commons Attribution Noncommercial License which permits any noncommercial use, distribution, and reproduction in any medium, provided the original author(s) and source are credited.

\section{References}

1. R.J. Dewhurst, D.A. Hutchins, S.B. Palmer, C.B. Scruby, Quantitative measurements of laser-generated acoustic waveforms. J. Appl. Phys. 53, 4064 (1982)

2. F.A. McDonald, Quantitative measurements of laser-generated acoustic waveforms. Appl. Phys. Lett. 54, 1504 (1989) 
3. M. Mesaros, O. Martínez, G.M. Bilmes, J.O. Tocho, Acoustic detection of laser induced melting of metals. J. Appl. Phys. 81, 1014 (1997)

4. H.K. Park, C.P. Grigoropoulos, W.P. Leung, A.C. Tam, A practical excimer laser-assisted cleaning tool for removal of surface contaminants. IEEE Trans. Compon. Packag. Manuf. Technol. A 17, 631 (1994)

5. A. Vogel, P. Schweiger, A. Frieser, M.N. Asiyo, R. Birngruber, Intraocular Nd:YAG laser surgery: Light-tissue interaction, damage range, and reduction of collateral effects. IEEE J. Quantum Electron. 26, 2240 (1990)

6. P. Teng, N.S. Nishioka, R.R. Anderson, T.F. Deutsch, Optical studies of pulsed-laser fragmentation of biliary calculi. Appl. Phys. B 42, 73 (1987)

7. A. Schilling, O. Yavas, J. Bischof, J. Boneberg, P. Leiderer, Absolute pressure measurements of a nanosecond time scale using surface plasmons. Appl. Phys. Lett. 69, 4159 (1996)

8. H.K. Park, D. Kim, C.P. Grigoropoulos, A.C. Tam, Quantitative measurements of laser-generated acoustic waveforms. J. Appl. Phys. 80, 4072 (1996)

9. F.V. Bunkin, A.A. Kolomensky, V.G. Mikhalevich, Lasers in Acoustics (Harwood Academic, Chur, 1991)

10. T. Varslot, S.E. Måsøy, Forward propagation of acoustic pressure pulses in 3D soft biological tissue. Model. Identif. Control 27, 181 (2006)
11. S.H. Ko, S.G. Ryu, N. Misra, H. Pan, C.P. Grigoropoulos, N. Kladias, E. Panides, G.A. Domoto, Laser induced short plane acoustic wave focusing in water. Appl. Phys. Lett. 91, 051128 (2007)

12. S.H. Ko, S.G. Ryu, N. Misra, H. Pan, C.P. Grigoropoulos, N. Kladias, E. Panides, G.A. Domoto, Laser induced plane acoustic wave generation, propagation and interaction with rigid structures in water. J. Appl. Phys. 104, 074103 (2008)

13. S.H. Ko, H. Pan, D.J. Hwang, S.G. Ryu, J. Chung, C.P. Grigoropoulos, D. Poulikakos, High resolution selective multilayer laser processing by nanosecond laser ablation of metal nanoparticle films. J. Appl. Phys. 102, 093102 (2007)

14. H.K. Park, C.P. Grigoropoulos, C.C. Poon, A.C. Tam, Modeling of pulsed laser heating of thin silicon films. ASME Trans. J. Heat Transfer 118 (1996)

15. Acoustics module user's guide, COMSOL Multiphysics (2006)

16. D.B. Bogy, F.E. Talke, Experimental and theoretical study of wave propagation phenomena in drop-on-demand ink jet devices. IBM J. Res. Dev. 28(3), 314-321 (1984)

17. K. Kwon, W. Kim, A waveform design method for high-speed inkjet printing based on self-sensing measurement. Sens. Actuators A 140, 75 (2007) 